\title{
In situ correction of mirror surface to reduce round-trip losses in Fabry-Perot cavities
}

\author{
G. Vajente $e^{1,2}$ \\ ${ }^{1}$ Istituto Nazionale di Fisica Nucleare, Sezione di Pisa, Largo B. Pontecorvo 3, 56124 Pisa, Italy \\ ${ }^{2}$ Currently at LIGO Laboratory, California Institute of Technology, Pasadena, California 91125 USA (vajente@caltech.edu) \\ Received 26 December 2013; revised 31 January 2014; accepted 31 January 2014; \\ posted 3 February 2014 (Doc. ID 203676); published 27 February 2014
}

\begin{abstract}
High-finesse resonant cavities play an important role in many metrology applications such as gravitational wave detectors. The performance of these cavities can be limited by round-trip losses (RTP) generated by light that is scattered by the mirror surface defects into higher-order modes that are close to resonance. In this paper we develop a detailed model of this effect and we study possible strategies to correct the mirror surface. We show that it is possible to restrict the correction to the combination of a reduced set of surface deformations that can be reproduced on the mirror using projected heating patterns. We show with an optical simulation that by acting on the cavity mirrors it is possible to reduce RTP to the large angle scattering limit. We also show that the optimal correction can be computed without any a priori knowledge of the mirror surface, but based only on measurements of the power stored inside the cavity, thus opening up the possibility of a simple implementation of the proposed algorithm. (C) 2014 Optical Society of America
\end{abstract}

OCIS codes: (120.2230) Fabry-Perot; (120.3180) Interferometry; (290.2648) Stray light; (220.1080)

Active or adaptive optics; (220.1000) Aberration compensation.

http://dx.doi.org/10.1364/AO.53.001459

\section{Introduction}

Interferometric gravitational wave (GW) detectors are designed to use $\mathrm{km}$ long resonant Fabry-Perot cavities in a Michelson configuration [1-3]. The sensitivity of these detectors is strongly linked to the quality of the resonant cavities. In particular, it is mandatory to keep the power losses inside the cavities as small as possible in order to profit from the resonant power build-up. In advanced GW detectors, the round-trip losses (RTL) are required to remain below a total of $75 \mathrm{ppm}$ per cavity [1]. Achieving low RTL is also relevant to build very high finesse cavities, which have a wide range of applications in optical metrology. One of these, which is of particular interest for the field of GW detection, is the implementation of filter cavities for squeezed vacuum sources [4].

$1559-128 X / 14 / 071459-07 \$ 15.00 / 0$

(C) 2014 Optical Society of America
RTL losses can originate from the surface defects of the cavity mirrors at different spatial frequencies. High-frequency defects scatter power at large angles, which is immediately lost from the cavity. Low and medium frequencies can couple the cavity fundamental mode into higher-order modes (HOMs) that can be partially resonant inside the cavity and therefore amplified. This effect extracts power from the main mode, increasing the RTL.

In a previous paper [5], a technique to reduce the RTL generated by the latter effect was proposed. From the known geometry of the resonant cavities it is possible to determine which HOM's are the closest to the resonance They are therefore the most important contributors to the RTL. The technique proposed consists of targeting only those defects of the mirror surface maps that generate these modes and compute a correction to cancel them. A technique for an in situ correction was proposed, projecting a thermal pattern onto the mirror's high reflectivity surface, which induces surface deformations through 
the thermo-elastic effect. It was demonstrated that the correction has a small amplitude and low spatial frequency since it aims only at canceling a particular set of HOM's.

In [5] the optimal correction map was computed assuming perfect knowledge of the mirror surface map. This assumption might not be valid however, since the amplitude of the defects that contribute to producing the total RTL might be very small and below current metrology capabilities. Moreover, additional deformations might be introduced by the way the mirrors are suspended and handled.

This paper continues the work described in [5]. As a first step, in Section 2 we recall how the mirror surface creates specific HOM's and in Section 3.A we study how they resonate inside a Fabry-Perot cavity. In advanced GW detectors it is preferable to avoid applying any thermal correction on the input mirror, since this optic is also traversed by the beam in transmission. The thermal correction would introduce an additional lensing outside of the FabryPerot cavity, introducing an additional burden on the thermal compensation system [6], which must correct for all aberrations inside the recycling cavity. The possibility of applying a correction only on the end mirror is discussed in Section 3.B.

We then study in more detail the creation of specific HOMs by particular mirror surface features. In Section $\underline{4}$ we show that a reduced number of mirror maps are sufficient to correct for the HOM's close to resonance. Based on these maps, an algorithm is developed to compute the optimal correction without any $a$ priori knowledge of the mirror surface figure but use only measurements of laser beam powers that will be easily available in GW detectors (Section 5).

Finally, in Section 6 we briefly discuss how the proposed technique can be extended to a full FabryPerot Michelson interferometer like those used in GW detectors.

In this paper the optical configuration of the Advanced Virgo arm cavities are taken as an example [1]; the length is of about $3 \mathrm{~km}$ and the two mirror radii of curvature are of 1420 and $1683 \mathrm{~m}$ for the input and end mirrors, respectively. Random mirror surface maps have been generated with a total root mean square roughness of abut $0.5 \mathrm{~nm}$ as described in [5]. Clearly the technique proposed here is applicable to the configuration of any other GW detector or high-finesse cavity.

\section{HOM Generations by a Mirror in the General Case}

In an ideal Fabry-Perot cavity, the two mirror surfaces are perfectly spherical and matched to the wavefront curvature of the resonant laser mode. The field at each point inside the cavity can be expanded in terms of the Hermite-Gauss modes corresponding to the cavity eigen-vectors $\mathrm{HG}_{m n}$ [7]:

$$
\Psi(x, y)=\sum_{m, n} \psi_{m n} \mathrm{HG}_{m n}(x, y)
$$

where we used $\Psi(x, y)$ to denote the full complex field as a function of the transverse coordinates and $\psi_{m n}$ to denote the coefficients of the field expansion in terms of the cavity eigen-modes.

In the real case, the surface of each mirror will deviate from the ideal surface by an amount that can be described as a function of the transverse coordinates $z(x, y)$, as shown in Fig. 1 . This deviation is typically much smaller than the laser wavelength, and the field resonant inside the cavity can still be described as a superposition of the ideal cavity eigen-modes. The effect of the imperfect mirror surface is therefore described as a coupling of the fundamental Gaussian mode $\mathrm{HG}_{00}$ to all higher-order Hermite-Gauss modes $\mathrm{HG}_{m n}$. In the approximation $|z(x, y)| \ll \lambda$ :

$$
\begin{aligned}
\Psi_{r}(x, y) & =r e^{-2 i k z(x, y)} \psi_{00} \mathrm{HG}_{00}(x, y) \\
& \simeq 2 i k r \sum_{m n} K_{m n} \psi_{00} \mathrm{HG}_{m n}(x, y),
\end{aligned}
$$

where $k=2 \pi / \lambda, \lambda$ is the wavelength of the light, $r$ is the mirror amplitude reflectivity, $\Psi_{r}$ is the field reflected by the mirror and $\psi_{00}$ is the amplitude of the fundamental mode circulating inside the cavity. The coupling coefficients for $m, n \neq 0$ are given by the following superposition integrals, again in the approximation $|z(x, y)| \ll \lambda$ :

$$
\begin{aligned}
K_{m n} & =\langle m n|z(x, y)| 00\rangle \\
& =\iint H G_{m n}^{*}(x, y) z(x, y) \mathrm{HG}_{00}(x, y) \mathrm{d} x \mathrm{~d} y .
\end{aligned}
$$

An important property of the values for $K_{m n}$ is that they are real. Indeed the imaginary part of the exponents of the two Gaussian modes cancel out and there remains only the product of the two Gaussian weights with the real-valued Hermite polynomials. Together with Eq. (2), this means that any surface deformation of the mirror can only couple the fundamental mode to HOMs with purely imaginary amplitudes.

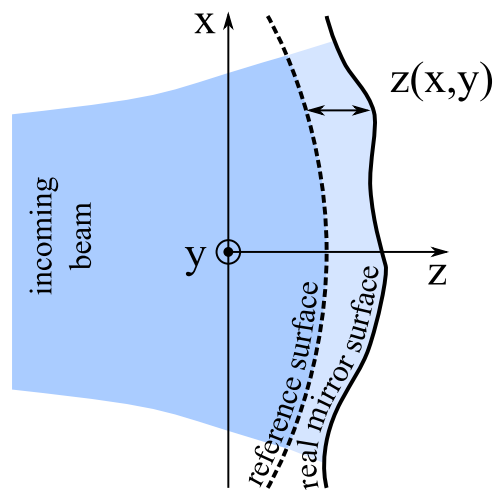

Fig. 1. Surface of any mirror can be described as the variation from a reference spherical surface, which is matched with the wavefront curvature of the incoming beam. The deviation is expressed as the distance along the propagation direction of the real surface from reference surface as a function of the transverse coordinates. 


\section{HOM Resonance inside a Fabry-Perot Cavity}

\section{A. RTL and HOM Content}

The situation is more complex when the aberrated mirror is part of a resonant Fabry-Perot cavity, since the HOM's that are generated by the surface defects propagate inside the cavity and might be resonant. Referring to Fig. 2, the input mirror of a Fabry-Perot cavity is assumed to be perfectly spherical, while the end mirror surface has a figure error that couples part of the fundamental $\mathrm{HG}_{00}$ mode to one selected $\mathrm{HOM} \mathrm{HG}_{m n}$. Since the space does not introduce additional couplings among modes, the steady-state field inside the cavity is characterized by the amplitudes of the fundamental mode $\psi_{i}$ and of the $\mathrm{HOM} \phi_{i}$ where the subscript $i$ refers to the different positions inside the cavity. The coupling can be described using Eq. (3):

$$
\varphi_{3}^{\prime}=\alpha \cdot i r_{2} \psi_{2},
$$

where $r_{2}$ is the reflectivity of the end mirror and for simplicity of notation we put $\alpha=2 \mathrm{kK}_{m n}$. To properly compute the effect on the fundamental mode of the mirror surface defects, it is necessary to also compute the reflectivity of the mirror for the $\mathrm{HG}_{00}$, to the leading order in $\alpha$. This can be easily computed from energy conservation and Eq. (4):

$$
\psi_{3}=\left(1-\frac{\alpha^{2}}{2}\right) \cdot i r_{2} \psi_{2}
$$

This term, however, is not the only one to the second order in $\alpha$ to contribute to the fundamental mode. Indeed it is possible that the HOM circulating inside the cavity couples back to the fundamental mode when reflected from the end mirror. The coupling coefficient is exactly the same as the one that governs the conversion of $\mathrm{HG}_{00}$ to $\mathrm{HG}_{n m}$, since it comes from the same superposition integral of Eq. (3):

$$
\psi_{3}^{\prime}=\alpha \cdot i r_{2}^{\prime} \varphi_{2} .
$$

This contribution must be added to that of Eq. (5) to obtain the correct fundamental mode amplitude in reflection of the end mirror. In this equation we have allowed the mirror reflectivity for the HOM to be different from that of the fundamental mode. This is

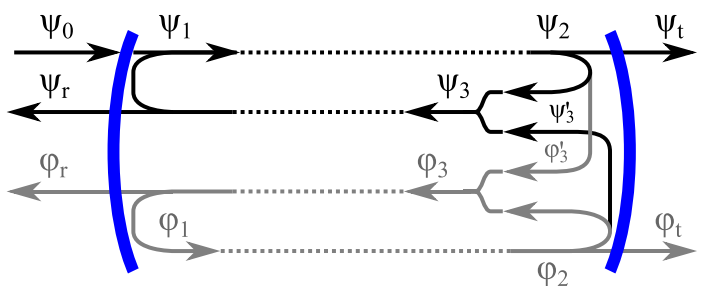

Fig. 2. Scheme of mode coupling in a resonant cavity. Here the input mirror is assumed to be perfect, while the end-mirror surface has some figure errors that couple part of the fundamental mode (in black) into HOMs (in gray). necessary as the HOM is much larger and therefore may suffer from higher clipping losses.

The fundamental mode acquires a phase $\Phi=$ $-k L+\Phi_{g}$ when propagating over the cavity length $L$. Here $\Phi_{g}$ indicates the cavity single-trip Gouy phase. The same is true for the HOM, with a different contribution from the Gouy phase shift $\Phi+(m+n) \Phi_{g}$. To simplify the notation we define $\Phi_{m n}=(m+n) \Phi_{g}$. Calling $r_{1}, t_{1}$ the reflectivity and transmissivity of the input mirror, the equations governing the steady state fields inside the cavity are

$$
\begin{aligned}
{\left[1-r_{1} r_{2}\left(1-\frac{\alpha^{2}}{2}\right) e^{-2 i \Phi}\right] \psi_{1}=} & t_{1} \psi_{0} \\
& +i r_{1} r_{2}^{\prime} \alpha e^{-2 i \Phi+i \Phi_{m n}} \varphi_{1}
\end{aligned}
$$

$$
\left[1-r_{1}^{\prime} r_{2}^{\prime}\left(1-\frac{\alpha^{2}}{2}\right) e^{-2 i \Phi-2 i \Phi_{m n}}\right] \varphi_{1}=i r_{1}^{\prime} r_{2} \alpha e^{-2 i \Phi+i \Phi_{m n}} \psi_{1}
$$

Equation (8) can be solved for the HOM field and the solution inserted in Eq. (7). With a bit of algebra one can find a complete expression for the fundamental mode amplitude inside the cavity, correct to second order in $\alpha$ :

$$
\psi_{1}=\frac{t_{1} \psi_{0}}{1-r_{1} r_{2} e^{-2 i \Phi}\left[1-\frac{\alpha^{2}}{2}\left(\frac{1}{2}+\frac{r_{1}^{\prime} r_{2}^{\prime} e^{-2 i \Phi+2 i \Phi_{m n}}}{1-r_{1}^{\prime} r_{2}^{\prime} e^{-2 i \Phi+2 i \Phi_{m n}}}\right)\right]} .
$$

The term inside the square brackets can be interpreted as a correction to the reflectivity of the end mirror. This correction is in general a complex number and therefore it can be described by a phase term $\delta \phi$, which shifts the resonance position of the fundamental mode and an amplitude term that accounts for additional RTL $\mathcal{L}$ depending on the resonance condition of the HOM:

$$
\begin{gathered}
\delta \phi=-\frac{\alpha^{2}}{2} \cdot \frac{1-r_{1}^{\prime 2} r_{2}^{\prime 2}}{1+r_{1}^{\prime 2} r_{2}^{\prime 2}} \cdot \frac{1}{1-\frac{2 r_{1}^{\prime} r_{2}^{\prime}}{1-r_{1}^{\prime} r_{2}^{\prime}} \cos \left(2 \Phi-2 \Phi_{m n}\right)}, \\
\mathcal{L}=\alpha^{2} \cdot \frac{1-r_{1}^{\prime 2} r_{2}^{\prime 2}}{1+r_{1}^{\prime 2} r_{2}^{\prime 2}} \cdot \frac{1}{1-\frac{2 r_{1}^{\prime} r_{2}^{\prime}}{1-r_{1}^{\prime} r_{2}^{\prime}} \cos \left(2 \Phi-2 \Phi_{m n}\right)}
\end{gathered}
$$

The shift of the resonant position is typically very small, and in any case it is compensated by the longitudinal control system of the cavity. Equation (11) shows analytically a result already found in [5] using an optical simulation. RTL losses can be increased due to conversion at one mirror of the fundamental mode into one HOM. Moreover, this effect is enhanced when the HOM becomes resonant inside the Fabry-Perot cavity, depending on the Gouy 
phase, which in turn depends on the mirror radii of curvature.

An important consequence of the result derived here is that when considering the RTL introduced by low and medium spatial frequencies, only the coupling to those modes that are close to resonance inside the Fabry-Perot cavities are relevant. In the case of an Advanced Virgo like geometry, those are eighth- and ninth-order modes.

In addition to the fundamental mode amplitude of Eq. (9), it is also possible to obtain from Eq. (7) to the leading order in $\alpha$, the amplitude of the HOM inside the cavity:

$$
\varphi_{1}=\alpha \frac{i r_{1}^{\prime} r_{2}^{\prime} e^{-2 i \Phi+2 i \Phi_{m n}}}{1-r_{1}^{\prime} r_{2}^{\prime} e^{-2 i \Phi+2 i \Phi_{m n}}} \cdot \frac{t_{1}}{1-r_{1} r_{2} e^{-2 i \Phi}} \psi_{0} .
$$

Comparing this equation with Eqs. (9) and (11) it appears that the RTL is proportional to the amplitude of the HOM generated inside the cavity, neglecting the additive factor $1 / 2$, which is negligible when the HOM is close to resonance. Therefore it is possible to study the effect of the HOM resonance on the RTL losses by computing the amplitude of the HOM inside the cavity. This is simpler, since it is sufficient to limit the calculation to the first order in the coupling coefficient $\alpha$.

Finally, Eq. (9) shows that the minimum RTL is obtained when the power inside the cavity is maximum, which in turn corresponds to the maximum of the power transmitted by the cavity.

\section{B. Cavity with Surface Errors on Both Mirrors}

In a real cavity both input and end mirror will have surface defects that can couple the fundamental mode to HOMs. Using the same notation as in the previous section, the amplitude of the HOM inside the cavity is given by

$$
\varphi_{1}=\frac{t_{1}}{1-r_{1} r_{2}} \cdot \frac{r_{1}^{\prime} r_{2}^{\prime}}{1-r_{1}^{\prime} r_{2}^{\prime} e^{2 i \Phi_{m n}}}\left(\alpha_{1}+e^{i \Phi_{m n}} \alpha_{2}\right) \psi_{0},
$$

where subscripts 1 and 2 refer respectively to the input and end mirrors and the cavity is assumed to be perfectly in resonance for the fundamental mode. The only difference between the two-mirror contributions is the phase of the HOM. Since the reference plane here is taken at the input mirror, the contribution coming from the end mirror has an additional phase due to the HOM propagation in the cavity. It is therefore clear that when both mirrors have defects, the HOM amplitude at the mirrors is no longer purely real but complex.

In addition to Eq. (13), it is possible to compute the HOM field amplitude in reflection of the cavity:

$$
\begin{aligned}
\varphi_{r}= & -r_{1}\left[\left(\alpha_{1}^{*}+\frac{e^{-2 i \Phi_{m n}}}{1-r_{1}^{\prime} r_{2}^{\prime} e^{-2 i \Phi_{m n}}} \frac{t_{1} t_{1}^{\prime} r_{2} r_{2}^{\prime}}{1-r_{1} r_{2}} \alpha_{1}\right)\right. \\
& \left.+\left(\frac{e^{-2 i \Phi_{m n}}}{1-r_{1}^{\prime} r_{2}^{\prime} e^{-2 i \Phi_{m n}}} \frac{t_{1} t_{1}^{\prime} r_{2} r_{2}^{\prime}}{1-r_{1} r_{2}} e^{-i \Phi_{m n}} \alpha_{2}\right)\right] \psi_{0} .
\end{aligned}
$$

Here the mixing of the contributions from the input and end mirrors is different since it was necessary to also consider the contribution coming from the prompt reflection of the beam impinging on the input mirror from outside the cavity, described by the complex conjugate-coupling coefficient $\alpha_{1}^{*}$. Comparing Eqs. (13) and (14) makes it clear that the only way to completely remove the HOM both inside the cavity and in reflection is to have $\alpha_{1}=\alpha_{2}=0$.

Starting from these computations we can better understand the viable strategies to correct mirror surface deformations. As pointed out in the Introduction, it would be preferable to correct by actuation on the end mirror only. From Eq. (13) it is possible to compute the HOM power, which is proportional to the RTL:

$$
\begin{aligned}
P_{\mathrm{HOM}}= & P_{0} \cdot \frac{4 k r_{1}^{\prime 2} r_{2}^{\prime 2}}{\left(1-r_{1}^{\prime} r_{2}^{\prime}\right)^{2}+4 r_{1}^{\prime} r_{2}^{\prime} \sin ^{2} \Phi_{m n}} \\
& \cdot\left[\alpha_{1}^{2}+\alpha_{2}^{2}+2 \alpha_{1} \alpha_{2} \cos \Phi_{m n}\right],
\end{aligned}
$$

where $P_{0}$ is the fundamental mode power stored inside the cavity. The addition of a correction map to the end mirror only $z_{2}(x, y) \rightarrow z_{2}(x, y)+c_{2}(x, y)$ has the effect of changing the coupling coefficient of the fundamental mode to the HOM:

$$
\begin{aligned}
\alpha_{2} & \rightarrow \alpha_{2}+k \iint H G_{m n}^{*}(x, y) c_{2}(x, y) \mathrm{HG}_{00}(x, y) \mathrm{d} x \mathrm{~d} y \\
& =\alpha_{2}+\beta_{2} .
\end{aligned}
$$

Equation (15) can be used to compute the minimum HOM power as a function of the correction coupling coefficient $\beta_{2}$. Assuming that it is possible to change arbitrarily the coupling coefficient (maintaining it real as required by the fact that no reflectivity correction is feasible), minimization of Eq. (15) yields the minimum achievable power in the $\mathrm{HOM}$ :

$$
P_{m n}=P_{00} \cdot \frac{4 k r_{1}^{\prime} r_{2}^{\prime 2}}{\left(1-r_{1}^{\prime} r_{2}^{\prime}\right)^{2}+4 r_{1}^{\prime} r_{2}^{\prime} \sin ^{2} \Phi_{m n}} \alpha_{1}^{2} \sin ^{2} \Phi_{m n} .
$$

This result shows that the minimum achievable power is directly linked to the cavity Gouy phase. In the extreme case when only the input mirror is aberrated and the correction is applied to the end mirror only, the maximum suppression of HOM power is given by $\sin ^{2} \Phi_{m n}$, which in the Advanced Virgo case is about $1 / 8$.

The conclusion is that in general, it is possible to only partially correct the RTL if one is forced to actuate on only one of the two mirrors. To achieve a complete removal of the HOM inside the cavity it is necessary to act on both mirrors.

\section{Surface Maps to Create Specific Modes}

We now move to the development of a strategy to selectively correct the relevant HOM's that are close to 
resonance. The examples shown here refer again to the Advanced Virgo case and to HOM's of order 8 and 9. It is interesting to understand which part of the surface figure contributes to the creation of a given mode. A study described in [8] showed that there is quite a close relationship between the mode order and the spatial frequency of the surface error which most efficiently couples energy into it. However, this study did not give any hint as to the exact shape of the mirror surface. In [9] a method was described to determine the surface deformation needed to efficiently couple Laguerre-Gauss modes into each other. That method could also be applied here in the case of Hermite-Gauss modes.

However, here we follow another method to determine the mode-creating maps. As suggested in [10], from Eq. (3) one could infer that the best way to reduce the coupling of the fundamental mode onto the set of HOM's under consideration is to minimize the projection of the surface $z(x, y)$ onto the set of functions $H G_{m n}^{*}(x, y) \mathrm{HG}_{00}(x, y)$. Therefore, one can guess that the components of the mirror surface that create those modes are combinations of the set of basis functions:

$$
M_{m n}=\mathrm{HG}_{m n} \mathrm{HG}_{00}^{*} .
$$

This set of possible correction maps is not orthogonal in the sense that if we apply the $i$-th map on a perfect mirror where a fundamental Gaussian mode is impinging, we obtain a coupling to all the eighthand ninth-order HOM's, not only the desired $i$-th one (see Fig. 3). It is however, possible to find an orthogonalization of this basis, which provides us with a set of maps, and each one generates a single HOM among the eighth- and ninth-order ones. The results are shown in Fig. 4 for the Advanced Virgo cavity configuration, corresponding to a beam radius on the input mirror of about $48 \mathrm{~mm}$. The set of maps

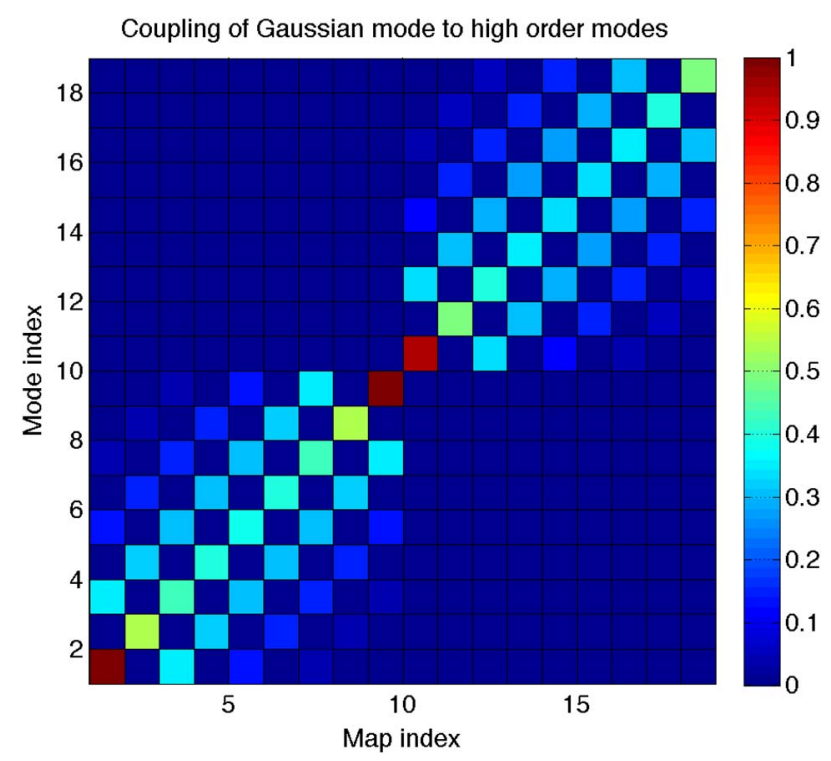

Fig. 3. Representation of the HOM coupling generated by the base maps described in Eq. (18). Each column corresponds to one of the maps. A Gaussian beam is reflected on the map and the amplitude of each HOM is represented by the color scale. The entire map has been rescaled setting the maximum to one.

for the end mirror are similar, but rescaled due to the different beam size.

The mode generating maps shown in Fig. 4 do not contain high spatial-frequency components. This means that they will be easily reproduced using an actuation system based on thermal correction, like the central heating residual aberration correction (CHRAC) system described in [5]. Moreover, the absence of high spatial-frequency components means that they will not increase the large angle scattering of the mirrors.

\section{HOM Correction Strategies in a Fabry-Perot Cavity}

To check the validity of the set of maps we have found, two random mirror maps have been created
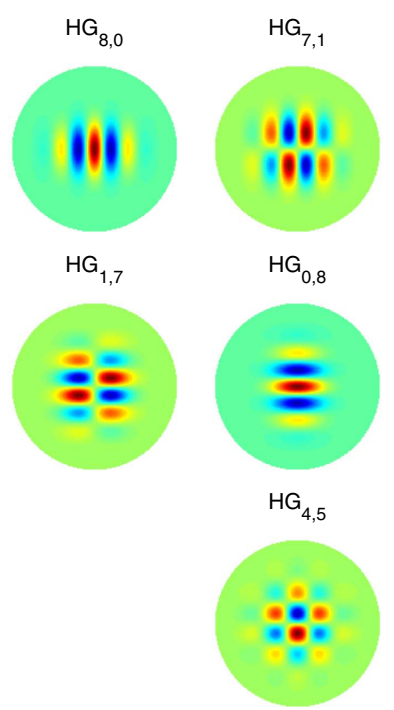

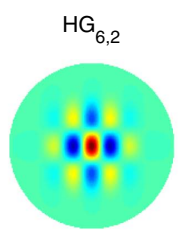

$H G_{9,0}$

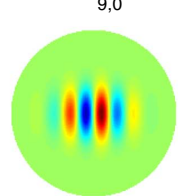

$\mathrm{HG}_{3,6}$

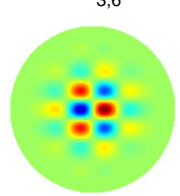

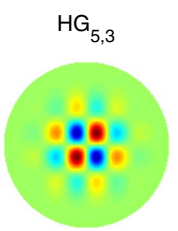

$H G_{8,1}$

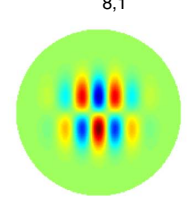

$\mathrm{HG}_{2,7}$

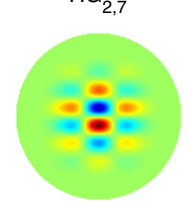

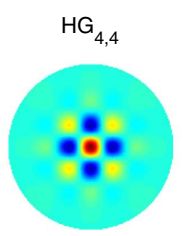

$\mathrm{HG}_{7,2}$

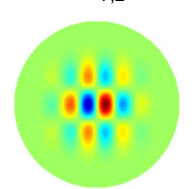

$H G_{1,8}$

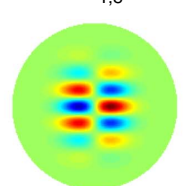

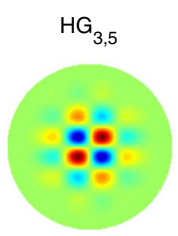

$H G_{6,3}$

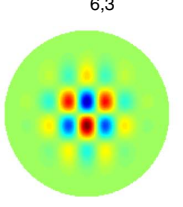

$H G_{0,9}$

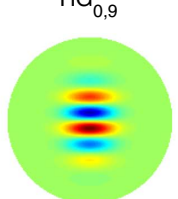

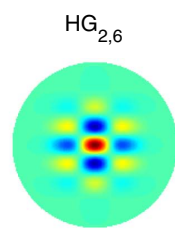

$H G_{5,4}$

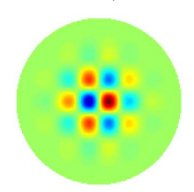

Fig. 4. Orthogonal set of maps that generates single eighth- or ninth-order mode when applied to a mirror. In this example the mirror has a diameter of $30 \mathrm{~cm}$ and the beam radius corresponds to about $48 \mathrm{~mm}$. 
and applied to the input and end mirrors of the cavity, as shown in the top row of Fig. 5. These maps are the same as those considered in [5]. The optimal correction map for each of the two mirrors can be computed as the linear combination of the mode-creating maps $M_{m n}$ that minimizes the total coupling to the eighth- and ninth-order HOM's power. Using the same procedure described in Section II of [9], we find the corrections maps shown in the bottom row of Fig. 5. Notably, these correction maps are the same as those obtained with a different strategy in [5]. We performed an FFT simulation of the entire cavity as described in [5]. Using the correction maps it is possible to reduce the RTL from 73.0 to $34.5 \mathrm{ppm}$ and correspondingly increase the cavity gain from 280.7 to 282.2 . These results show that the set of mode generating maps are complete; they are sufficient to completely remove the generation of eighthand ninth-order modes inside the cavity.

As explained in the Introduction, it is important to develop a procedure to find the optimal map without any $a$ priori knowledge of the mirror surface maps. Since RTL is linked to the power stored inside the cavity as shown before, one can imagine implementing an optimization algorithm that tries to maximize the power stored inside the cavity, changing the correction maps applied to the two mirrors. The mode creating maps discussed in the previous section can be used to reduce the dimensionality of the search space from the number of pixels of the thermal correction system (of the order of 100) to the number of HOMs that are relevant, i.e., close to resonance. In the Advanced Virgo case the eighth- and ninth-order modes were considered, giving a total of $N=19$ per mirror. This would already provide a significant reduction in the search complexity.
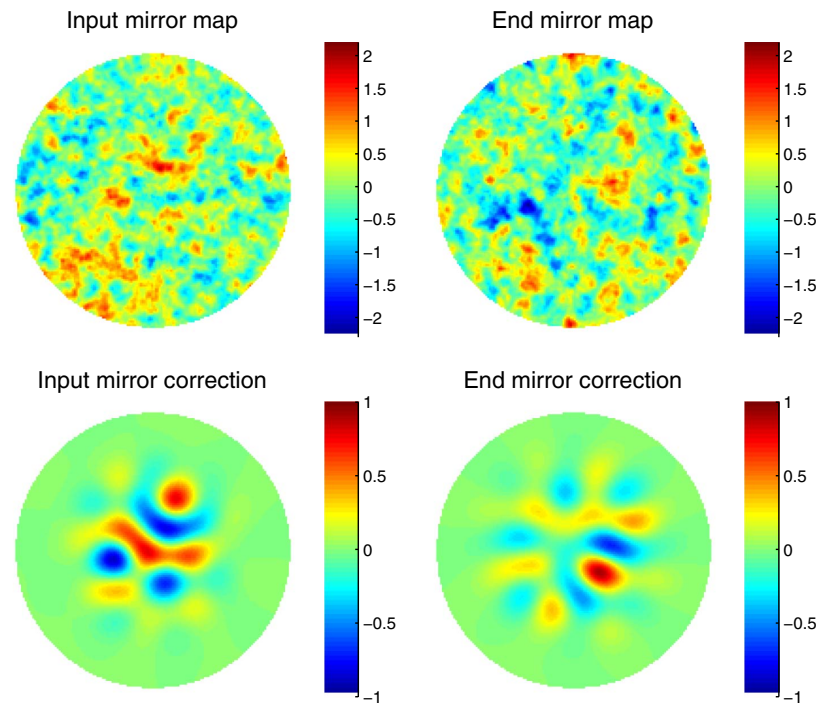

Fig. 5. Top row, randomly generated mirror maps for the input (left) and end (right) mirrors of a Fabry-Perot resonant cavity with Advanced Virgo geometry. Bottom row, correction maps computed as linear combination of the mode generating maps of Fig. $\underline{4}$. The mirror diameter is $30 \mathrm{~cm}$.
However, the orthogonality of the mode creating maps suggests that it is possible to consider only one mode at a time. The optimal correction can therefore be found implementing $N$ subsequent searches, each for any of the modes under consideration. For each mode, the optimal amplitude of the maps for both input and end mirror corrections must be found simultaneously, as a consequence of the discussion of Section 3.B. Therefore, the algorithm to find the two optimal mirror corrections boils down to $N$ sequential bi-dimensional searches. The complexity of this approach is much lower than that of a full search.

To test this idea, we implemented it with the same optical simulation described above. After the $N$ searches are concluded, the RTL are reduced to $34.9 \mathrm{ppm}$ and the cavity gain is increased to 282.28 . It is interesting to note that the maps found in this way (see Fig. 6) are different from those found with the corrections computed from the mirror-map knowledge. This is reflected in the small difference in the final RTL value. The discrepancy is explained by the fact that our algorithm tries to maximize the cavity power, which depends mainly but not only on the eighth- and ninth-order modes. Both the mirror maps and the correction maps introduce couplings into other HOM's, that in turn contributes to the RTL. These additional couplings were not taken into account in the orthogonalization process described in Section 4 , which was restricted to the eighth and ninth-order modes.

It is possible to repeat the series of sequential searches, starting this time from the correction maps found at the first trial. As expected, this second iteration helped tackle the non-orthogonality and improved the performance. RTL was reduced to $31.2 \mathrm{ppm}$ and the cavity gain increased to 282.41 . This is compatible with the expected minimum RTL given by large-angle scattering. Both results are better than those obtained with the correction computed from the mirror surface maps. This is again understood since in the latter case the goal was only the minimization of eighth- and ninth-order modes without any knowledge of the cavity gain.

\section{Application to GW Detectors}

The application of the algorithm proposed in the previous section should not pose major difficulties in a
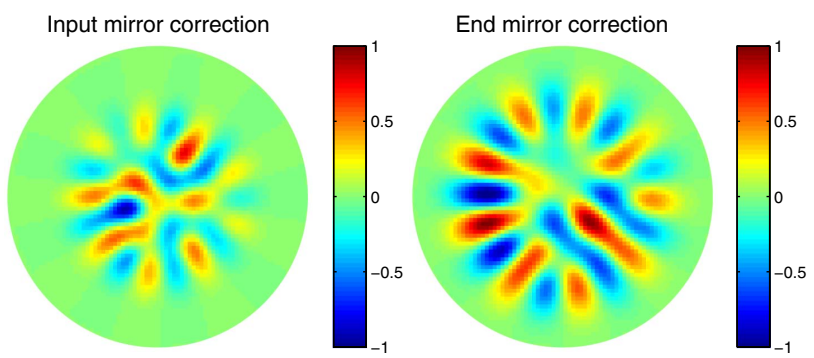

Fig. 6. Mirror correction found with the bi-dimensional sequential search algorithm described in the text. These should be compared with those shown in Fig. $\underline{5}$ obtained from the knowledge of the mirror surface maps. 
real cavity like those used in GW-interferometric detectors. The main limitation to the time needed to perform the searches is given by the thermal response of the mirrors to changes in the applied correction, which can be of the order of tens of minutes. However, since the correction depends on defects on the mirror surface, which do not change over time, the optimal correction must be computed only once and does not need to be adjusted in real-time. To speed up the searches it is possible to take advantage of the measurable time response of the correction [11] and implement model predictive control systems [12].

In GW detectors the two Fabry-Perot cavities are coupled by the presence of the power recycling mirror [13]. If the RTL of one of the two cavities is reduced, the recycling gain of the interferometer is increased, resulting in more power at the input of both cavities. This means that it is not possible to operate the searches in parallel in the two cavities, since it would be impossible to tell in which cavity the improvement had originated. This problem, however, can be overcome by operating the system without power recycling or simply correcting one cavity at a time.

\section{Conclusions}

The reduction of RTL in high finesse cavities is a crucial step to reach the goal of GW detectors and other high-sensitivity metrology experiments. In this paper we described in detail how RTL is increased by mirror surface defects that create HOMs that are close to resonance. Continuing the work of [5] we showed that it is possible to find the optimal correction that reduces the RTL to the large angle scattering level, without any a priori knowledge of the mirror maps. This opens up the possibility of the application of the proposed CHRAC technique to second-generation GW wave detectors and filter cavities for squeezed vacuum sources, where the mirror defects are expected to be too small to be measured in a reliable way with state-of-the-art metrology technology. In advanced GW detectors it would be preferable to apply the proposed thermal correction on the end mirror only. However, we demonstrated that in this way it is not possible to completely remove the detrimental effect on RTL due to HOMs resonance.

Since the proposed algorithm does not need any additional special hardware to reconstruct the correction, we expect that its application to high-finesse cavities, in particular in advanced GW detectors, could be rather simple.

The author is especially grateful to R. Day (European Gravitational Observatory) for useful discussions and comments. This research activity has been partially supported by Regione Toscana (Italy) through the program POR CreO FSE 2007-2013 of the European Community, within the project n. 18113 (ISAV). LIGO was constructed by the California Institute of Technology and Massachusetts Institute of Technology with funding from the United States National Science Foundation under grant PHY0757058 .

\section{References}

1. The Virgo Collaboration, "Advanced Virgo Baseline Design," VIR-027A-09, https://tds.ego-gw.it/ql/?c=6589.

2. G. M. Harry, for the LIGO Scientific Collaboration, "Advanced LIGO: the next generation of gravitational wave detectors," Class. Quant. Grav. 27, 084006 (2010).

3. K. Kuroda, "Large-scale gravitational wave telescope (LCGT)," Int. J. Mod. Phys. D 20, 1755-1770 (2011).

4. P. Purdue and Y. Chen, "Practical speed meter designs for quantum nondemolition gravitational-wave interferometers," Phys. Rev. D 66, 122004 (2002).

5. R. A. Day, G. Vajente, M. Kasprzack, and J. Marque, "Reduction of higher order mode generation in large scale gravitational wave interferometers by central heating residual aberration correction (CHRAC)," Phys. Rev. D 87, 082003 (2013).

6. A. Rocchi, E. Coccia, V. Fafone, V. Malvezzi, Y. Minenkov, and L. Sperandio, "Thermal effects and their compensation in Advanced Virgo,” J. Phys. Conf. Ser. 363, 012016 (2012).

7. E. Siegman, Lasers (University Science, 1986).

8. W. Winkler, R. Schilling, K. Danzmann, J. Mizuno, A. Ridiger, and K. A. Strain, "Light scattering described in the mode picture," Appl. Opt. 33, 7547-7550 (1994).

9. G. Vajente and R. A. Day, "Adaptive optics sensing and control technique to optimize the resonance of the Laguerre-Gauss 33 mode in Fabry-Perot cavities," Phys. Rev. D 87, 122005 (2013).

10. T. Hong, J. Miller, H. Yamamoto, Y. Chen, and R. Adhikari, "Effects of mirror aberrations on Laguerre-Gaussian beams in interferometric gravitational-wave detectors," Phys. Rev. D 84, 102001 (2011).

11. A. Haber, A. Polo, S. Ravensbergen, H. P. Urbach, and M. Verhaegen, "Identification of a dynamical model of a thermally actuated deformable mirror," Opt. Lett. 38, 3061-3064 (2013).

12. M. Morari, C. E. Garcia, and D. M. Prett, "Model predictive control: theory and practice a survey," Automatica 25, 335348 (1989)

13. M. Ando, K. Arai, K. Kawabe, and K. Tsubono, "Demonstration of power recycling on a Fabry-Perot-type prototype gravitational wave detector," Phys. Lett. A 248, 145-150 (1998). 